\title{
Thermal response and optical absorptance of metals under femtosecond laser irradiation
}

\author{
Anatoliy Y. Vorobyev, Chunlei Guo
}

The Institute of Optics, University of Rochester, Rochester, USA; vorobyev@optics.rochester.edu, guo@optics.rochester.edu

Received 15 March 2011; revised 10 April 2011; accepted 27 April 2011.

\begin{abstract}
A detailed study on correlation between residual thermal response of a sample and its optical absorptance change due to laser-induced surface structural modifications in multi-shot femtosecond laser irradiation is performed. Experiments reveal an overall enhancement for residual thermal coupling and absorptance in air. Surprisingly, residual thermal coupling in air shows a non-monotonic dependence on pulse number and reaches a minimum value after a certain number of pulses, while these behaviors are not seen in absorptance. In vacuum, however, both suppression and enhancement are seen in residual energy coupling although absorptance is always enhanced. From these observations, it appears that air plasma plays a dominant role in thermal coupling at a relatively low number of applied pulses, while the formation of craters plays a dominant role at a high number of pulses.
\end{abstract}

Keywords: Femtosecond Laser; Ablation; Residual Energy; Absorptance; Surface Structures

\section{INTRODUCTION}

Many applications of femtosecond lasers, such as high-precision materials machining [1,2], nanotechnology [3-7], modification of optical properties of materials [8-12], thin film deposition [13], modification of wetting properties of solids [14-17], producing diamond-like materials [18], laser plasma thrusters [19], and biomedicine $[17,20]$, are based on laser ablation of solids. Although femtosecond laser ablation have been extensively studied in the past [21-27], many of the physical mechanisms remain unclear. For example, recent studies have shown that the residual thermal energy in an irradiated metal sample abruptly increases following both single-pulse and pulse-train femtosecond laser ablation in a gas medium when the laser fluence is above a certain threshold [28,29]. In the case of pulse-train ablation [29], it was found that one contributing factor to the enhanced thermal coupling following a large number of applied pulses is an increase of sample absorptance due to surface structural modifications. However, we have shown that the surface modifications alone cannot fully explain the enhanced thermal energy deposition [29]. To further study the effects of the enhanced thermal coupling to metals, in this paper, we perform a shot-to-shot detailed study on how residual energy correlates to the absorptance change due to surface structural modifications. We observe an overall enhancement for residual thermal coupling and absorptance in air. Surprisingly, residual thermal coupling in air shows a non-monotonic dependence on pulse number and reaches a minimum value after a certain number of pulses, while these behaviors are not seen in absorptance. In vacuum, however, both suppression and enhancement are seen in residual energy coupling although absorptance is always enhanced. To explain these observations, we suggest that air plasma plays a dominant role in thermal coupling at a relatively low number of applied pulses while the formation of craters plays a dominant role at a high number of pulses.

\section{EXPERIMENTAL SETUP}

The laser used in the experiment is an amplified Ti:sapphire system generating 65 -fs pulses of about 1.5 $\mathrm{mJ} /$ pulse at a $1-\mathrm{kHz}$ repetition rate with the central wavelength at $800 \mathrm{~nm}$. Our experimental setup is shown in Figure 1. To produce ablation, the laser beam is focused onto a platinum $(\mathrm{Pt})$ bulk sample at normal incidence. The Pt sample is mechanically polished before experiments. The number of laser pulses, $N$, delivered to the sample is controlled by a fast electromechanical shutter.

Following pulse irradiation, some directly absorbed laser energy will be carried away by the ablated material, while a fraction of the absorbed pulse energy will remain in the surface layer of the sample, dissipate into the bulk sample due to heat conduction, and remain in the sample as residual thermal energy. The remaining energy fol- 


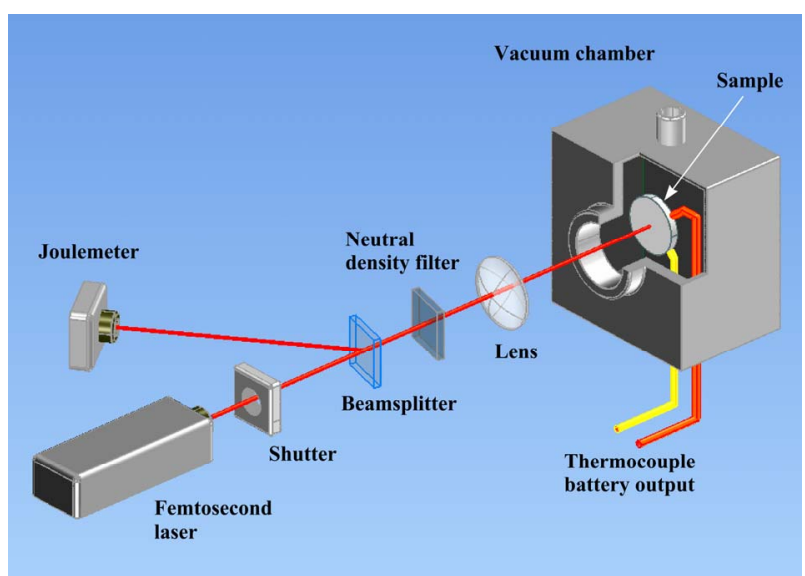

Figure 1. Experimental setup for studying both residual thermal coupling and absorptance change of a metal due to surface structural modifications following pulse irradiation.

lowing irradiation will cause the bulk sample temperature to rise. Furthermore, there can be indirect mechanisms contributing to the residual heating of the sample, such as re-deposition of the hot ablated material back onto the sample and exothermic chemical reactions. To characterize the total residual thermal energy coupling to the sample, we define a residual energy coefficient (REC), $K$, as $K=E_{R} / E_{I}$, where $E_{R}$ is the total residual energy remaining in the sample following pulse irradiation and $E_{I}$ is the pulse energy incident onto the sample. To measure $E_{R}$, we apply a calorimetric technique that has been described in our previous studies [28,29]. Briefly, we measure the bulk temperature rise $\Delta T_{R}$ with a thermocouple battery following ablation and determine $E_{R}$ calorimetrically as $E_{R}=m C \Delta T_{R}$, where $m$ is the mass and $C$ is the known specific heat capacity of the sample. To measure $E_{I}$, a fraction of the incident pulse energy is split off by a beamsplitter and diverted to a pyroelectric joulemeter. This calorimetric technique can also be used to study the change of the sample absorptance due to laser-induced surface modifications by applying the following procedure that has also been described in our previous work [9]. To determine the absorptance of a damaged material following pulse irradiation, we attenuate the laser fluence with neutral density filters to a level much below the ablation threshold and apply the same calorimetric technique as used in determining REC. Briefly, after ablation we will irradiate the ablated spot using a train of low-fluence laser pulses that will not produce further surface modifications. The absorbed fraction of this low-fluence pulse train energy, $E_{A}$, will dissipate into the sample due to heat conduction and cause the bulk temperature of the whole sample to rise by $\Delta T_{A}$. We measure $\Delta T_{A}$ with the thermocouple battery and determine calorimetrically the energy $E_{A}$ absorbed by the sample. Having also measured the total energy of the low-fluence pulse train, $E_{I T}$, the absorptance of the sample following laser ablation is determined as $A=$ $E_{A} / E_{I T}$. Thus, our calorimetric technique allows us to measure both REC and absorptance of the ablated sample and to determine the relationship between REC and absorptance. Our experimental procedure is as follows. We apply the first laser pulse to an undamaged Pt sample with fluence above the ablation threshold and determine REC. We then attenuate the beam with the neutral density filters and measure the absorptance of the irradiated spot. Next, we remove the filters, apply the second high-fluence pulse, and determine REC; we then reinsert the filters and measure the absorptance $A$ following two-pulse ablation. This measurement is repeated at the same sample spot, so we can determine both REC and $A$ as a function of pulse number $N$. Our experiment is carried out in both 1-atm air and in a vacuum at a base pressure of $6 \times 10^{-3}$ Torr. The number of applied laser shots is varied from 1 to 1000 . Following pulse irradiation, the irradiated spots are examined under a scanning electron microscope (SEM).

\section{EXPERIMENTAL RESULTS}

Following femtosecond laser irradiation, the residual energy coefficient $K(N)$ and low-fluence absorptance $A(N)$ are studied as functions of the number of applied laser pulses, $N$, at single-pulse laser fluence of $F=9.0$ and $3.6 \mathrm{~J} / \mathrm{cm}^{2}$ in both air and vacuum. The single-pulse ablation threshold $F_{a b l}$ for the mechanically polished $\mathrm{Pt}$ surface is determined to be $F_{a b l}=0.15 \mathrm{~J} / \mathrm{cm}^{2}$. The number of pulses required to drill through a 0.25 -mm-thick Pt sample in air is determined to be 920 and 1530 at $F=$ 9.0 and $3.6 \mathrm{~J} / \mathrm{cm}^{2}$, respectively. This gives an average ablation rate of 272 and $163 \mathrm{~nm} /$ pulse for the two fluence levels. As a reference, we also measure the low-fluence absorptance $A_{0}(N)$ for an undamaged surface at fluence much below the ablation threshold. This $A_{0}(N)$ is shown with the cross symbols in Figure 2.

One can see that $A_{0}$ is a constant value of about 0.34 and is independent of the number of applied shots, indicating that this laser fluence is indeed low enough and no heat accumulation effects are involved. This value of $A_{0}$ ( $\left.\sim 0.34\right)$ agrees with the table value of the absorptance for a mechanically polished Pt at $\lambda=800 \mathrm{~nm}$ [30]. The dependences of REC and absorptance on the number of ablation shots at $F=9.0 \mathrm{~J} / \mathrm{cm}^{2}$ in 1-atm air are shown in Figure 2. We notice two distinct regions in the behavior of $K(N)$ and $A(N)$. For $N<12$, REC decreases with $N$ while for $N>12$, REC increases with $N$. An important feature in the behavior of REC is that the highest REC value $(K=0.76)$ is reached by only one single pulse ablation although the absorptance of the undamaged surface before the first pulse irradiation is the lowest $(A=$ 


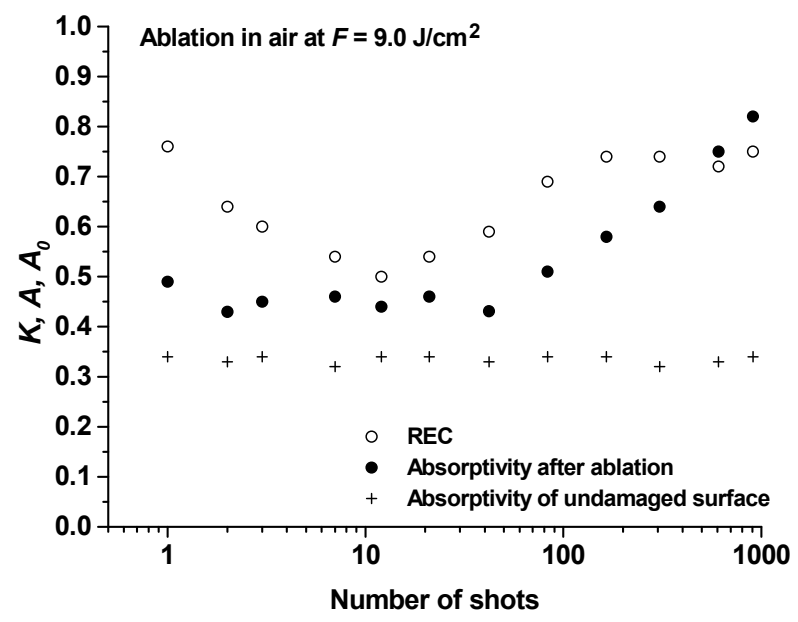

Figure 2. The pulse number dependences of residual energy coefficient and absorptance for platinum following ablation at $F=9.0 \mathrm{~J} / \mathrm{cm}^{2}$ in 1 -atm air.

0.34). Following the second pulse irradiation, REC decreases from 0.76 to 0.64 although the sample absorptance $(A=0.5)$ before the second pulse is much higher than the undamaged surface value. For $2<N<12$, REC decreases while the absorptance remains virtually the same. These data clearly show that for low $N$ the enhanced absorptance compared to the undamaged surface is not the dominant factor for the enhanced residual thermal heating. For $N>20$, REC starts to increase with $N$ and reaches a saturation level of about 0.75 at $N \approx 200$. Therefore, in the range of $N$ studied in this experiment, about $50 \%-75 \%$ of incident pulse energy remains in the sample as residual thermal energy (thermal load). The $A(N)$ data in Figure 2 show that the absorptance enhances from 0.34 (undamaged surface) to 0.5 following single-pulse ablation, then remains approximately constant for $N<30$. To determine the surface structures associated with the absorptance enhancement, we take SEM images of the sample surface. As shown in Figure 3, the enhanced surface absorptance mainly results from the laser-induced micro- and nano-roughness [9]. With further increasing $N$, we observe the formation of an ablation-induced crater (see Figure 4(a)) and both REC and absorptance increase as the cavity develops. From Figure 2, we can see that $A$ surpasses $K$ when $N>600$.

The behaviors of $A_{0}(N), A(N)$, and $K(N)$ measured in a vacuum are shown in Figure 5. We can see that the low-fluence absorptance $A_{0}(N)$ of an undamaged surface is about the same as the value measured in air. The overall behavior of $A(N)$ in vacuum is similar to that in air, although the absorptance enhancement due to surface structural modifications is greater in vacuum compared to that in air. However, the dependence of $K$ on $N$ in vacuum is very different from that in air. We can see that

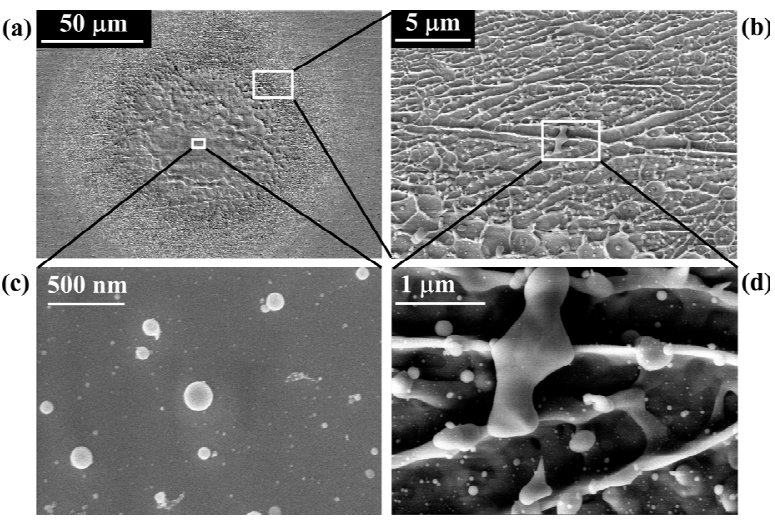

Figure 3. SEM images of the surface morphology of the irradiated spot after one-shot ablation of platinum at $F=$ $9.0 . \mathrm{J} / \mathrm{cm}^{2}$ in 1-atm air: (a) the general view of the ablated spot; (b) micro- and nano-structural features on the periphery of the ablated spot; (c) spherical nanoparticles re-deposited in the central area of the ablated spot; (d) magnified view of the peripheral surface structures shown in (b). The diameter of the irradiated spot is about $100 \mu \mathrm{m}$.
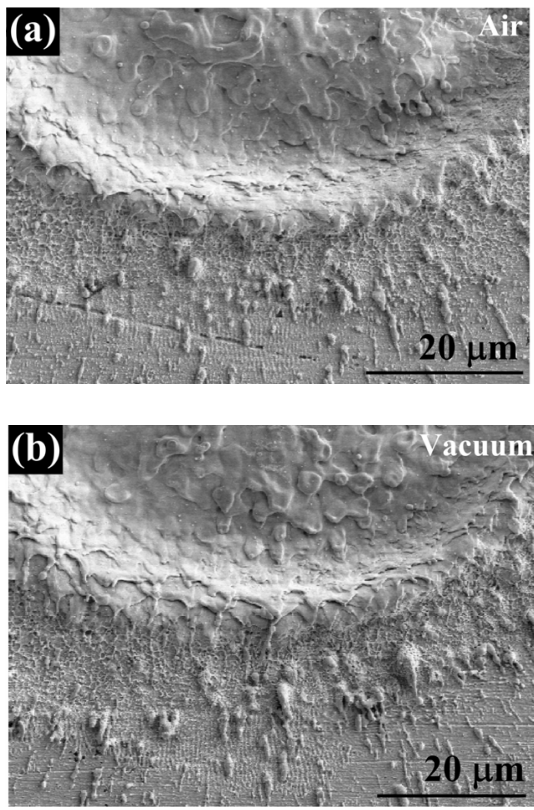

Figure 4. SEM images showing the onset of the cavity formation following 20 -shot ablation at $F=9.0 \mathrm{~J} / \mathrm{cm}^{2}$ in air (a) and in vacuum (b).

following the first pulse ablation, REC reaches a value of 0.15 in vacuum, well below the value of absorptance of the undamaged surface. This low REC value in vacuum contrasts with a much higher REC value we observe in air ( $K=0.76$ higher than the absorptance value). For $N$ between 2 and about 20 shots, REC remains to be nearly constant at 0.15 despite the absorptance has increased from 0.44 to 0.74 . For $N>20$, REC increases significantly and reaches a value of about 0.85 at a large 


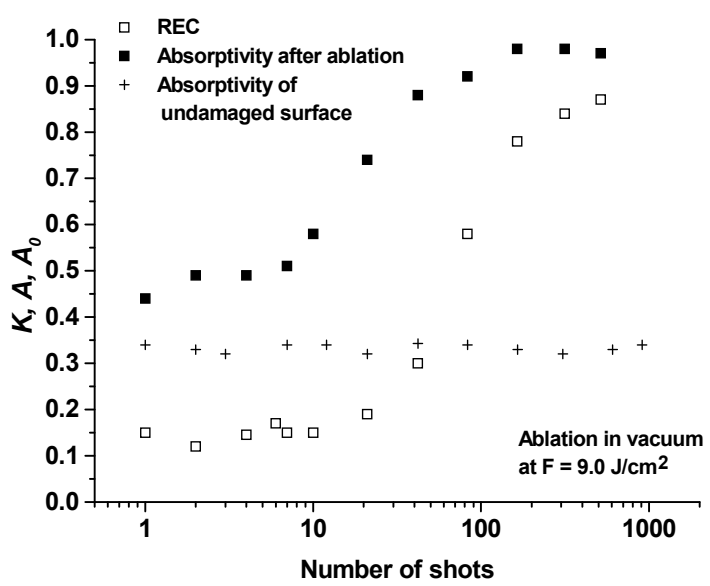

Figure 5. The pulse number dependences of residual energy coefficient and absorptance following ablation at $F$ $=9.0 \mathrm{~J} / \mathrm{cm}^{2}$ in a vacuum at a base pressure of $6 \times 10^{-3}$ Torr.

$N$. In the entire range of $N$, we notice that $K<A$ for ablation in vacuum, which contrasts with the relationship of $K>A$ in air. We also take SEM images of surface morphology following ablation in vacuum under the same ablation conditions as in air and some representative images are shown in Figure 6. A comparative analysis of the images taken for ablation in air versus in vacuum shows that the central area of the ablated spot in vacuum is free of redeposited nanoparticles (compare Figure 6(c) with Figure 3(c)) although a small amount of redeposited particles may be observed on the peripheral area of the ablated spot. Figure 5 shows that both $K(N)$ and $A(N)$ begin to increase significantly for $N$ in the range of $10-20$ shots in vacuum. The SEM study reveals that these enhancements correlate with the cavity formation (see Figure 4(b)). Therefore, for ablation both in air (Figures 2 and 4(a)) and in vacuum (Figures 5 and 4(b)), the onset of the significant REC and absorptance enhancements occurring at $N \approx 10$ - 30 corresponds to the formation of a crater.

To study the effects of laser fluence on REC and absorptance in multipulse ablation, we also measure $K(N)$ and $A(N)$ following ablation at lower fluence of 3.6 $\mathrm{J} / \mathrm{cm}^{2}$. The obtained results are plotted in Figures 7 and 8. The comparison of these lower-fluence data with the higher-fluence data in Figures 2 and 5 shows that the lower laser fluence mostly affects $K(N)$, and we observe the relationship of $K<A$ at $F=3.6 \mathrm{~J} / \mathrm{cm}^{2}$ as opposed to $K>A$ at $F=9.0 \mathrm{~J} / \mathrm{cm}^{2}$. To further understand the effects of laser fluence on residual thermal coupling, we measure the fluence dependence of $K$ following single-pulse $(N=1)$ ablation in air, neon, and vacuum by irradiating a fresh spot on the Pt sample after each shot. The behavior of $K(F)$ following a single femtosecond pulse ablation of an undamaged surface is shown in Figure 9. We see that

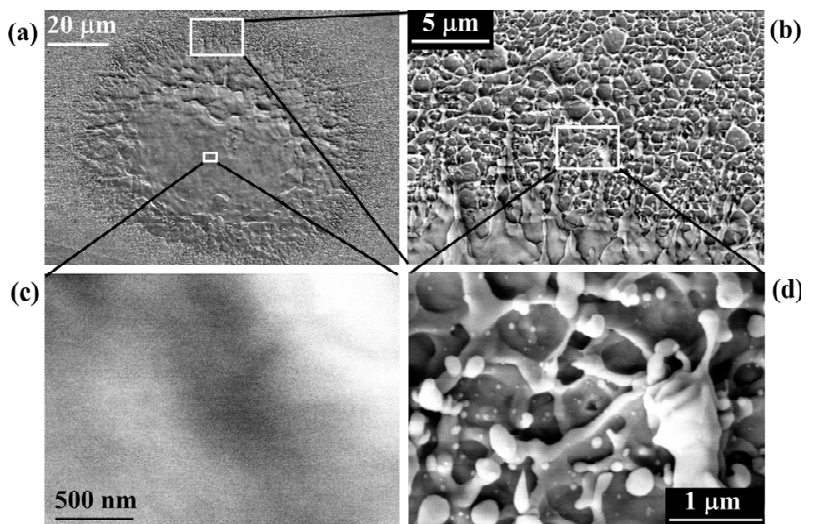

Figure 6. SEM images of surface morphology of the irradiated spot after one-shot ablation of platinum at $F=9.0 \mathrm{~J} / \mathrm{cm}^{2}$ in vacuum: (a) the general view of the ablated spot; (b) microand nano-structural features on the periphery of the ablated spot; (c) the magnified view of the central area of the ablated spot (there is no re-deposited nanoparticle as compared to Fig. 3(c)); (d) the magnified view of the peripheral surface structures shown in (b). The diameter of the irradiated spot is 100 $\mu \mathrm{m}$

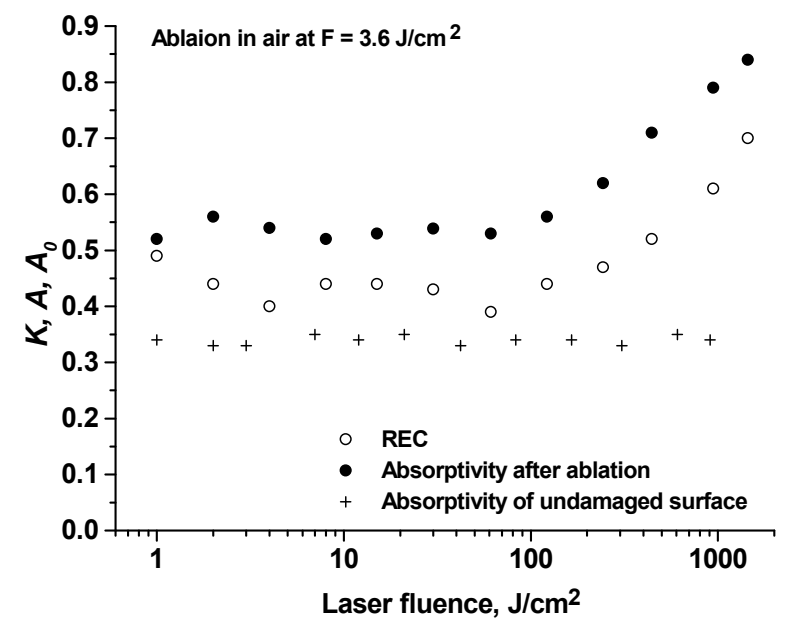

Figure 7. The pulse number dependences of residual energy coefficient and absorptance following ablation at $F=$ $3.6 \mathrm{~J} / \mathrm{cm}^{2}$ in 1 -atm air.

the three curves are virtually overlap on each other for $F$ $<1.2 \mathrm{~J} / \mathrm{cm}^{2}$. However, these three curves start to branch out for $F>1.2 \mathrm{~J} / \mathrm{cm}^{2}$, i.e., REC increases both in air and neon but decreases in vacuum. This measurement shows that the specific type of ambient gas plays very little role in affecting REC, but the presence of an ambient gas is the dominant factor for the enhanced REC. To further understand the ambient gas pressure effects on residual thermal energy deposition, we measure the pressure dependence of $K$ and the data are shown in Figure 10. We can see that REC decreases as the ambient air pressure is lowered. This pressure dependence becomes insignificant in the low-pressure range, i.e., $P<0.03$ Torr. 


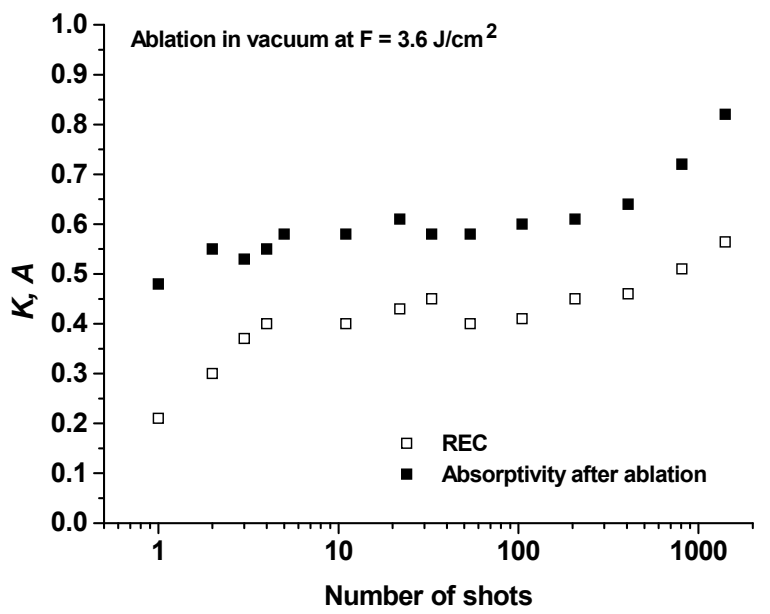

Figure 8. The pulse number dependences of residual energy coefficient and absorptance following ablation at $F=3.6$ $\mathrm{J} / \mathrm{cm}^{2}$ in a vacuum at a base pressure of $6 \times 10^{-3}$ Torr.

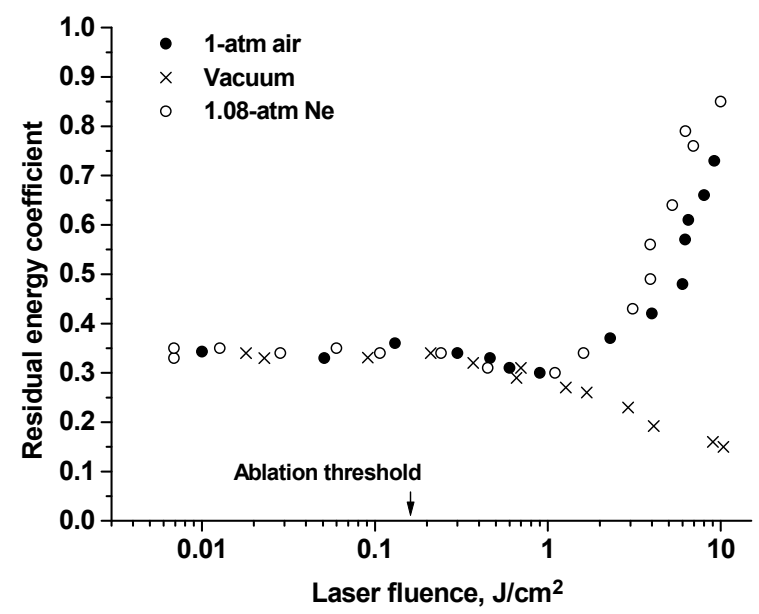

Figure 9. Residual energy coefficient for platinum as a function of laser fluence in 1-atm air, 1.08-atm $\mathrm{Ne}$, and a vacuum at a base pressure of $6 \times 10^{-3}$ Torr.

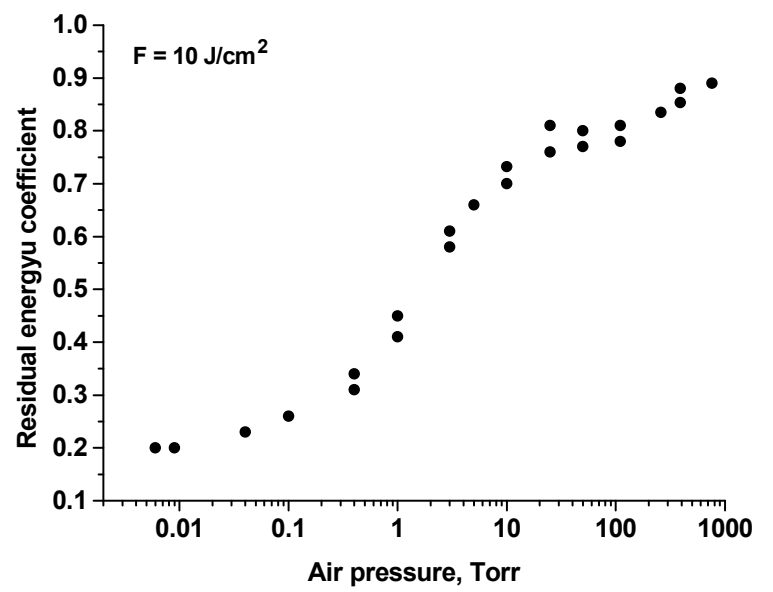

Figure 10. Residual energy coefficient for platinum as a function of air pressure at $F=10 \mathrm{~J} / \mathrm{cm}^{2}$.

\section{DISCUSSIONS}

Thermal response of metals following high-fluence laser irradiation has been studied in the past using microsecond [31] and nanosecond [32] laser pulses, and enhanced heat deposition in metals has been found in those experiments when plasmas are generated. To explain those enhanced thermal energy coupling, it has been suggested that energy transferring to a metal sample from laser-induced plasmas plays a key role [31]. Previously, we have found that the behavior of residual thermal energy coupling shows a similar general trend between nanosecond- and femtosecond-laser pulse ablation [28], and this suggests that the physical mechanism used to explain the long-pulse REC enhancement may also account for the behaviors of residual heating in femtosecond laser ablation. In general, two types of plasmas can be produced during a femtosecond laser pulse: 1) solid-density plasma in the skin layer of the sample and 2) ambient gas plasma. Solid-density plasma can be produced in both air and vacuum; in our experiment, however, the enhanced thermal coupling occurs only in air and therefore, we believe the solid-density plasma does not play a key role in the enhanced energy coupling. Therefore, we will subsequently focus our discussion on the effects of ambient air plasma.

The experimental data shown in Figure 2 are obtained with an intensity of about $10^{14} \mathrm{~W} / \mathrm{cm}^{2}$. At this high intensity, direct ionization of the ambient air during the femtosecond laser pulse easily occurs by means of multiphoton and tunneling ionization even without the presence of a metal sample [33]. In the presence of a metal surface, the threshold of air ionization in front of the metal surface can be significantly reduced due to: 1) air exposing to the additional laser light reflected from the metal surface besides the incident light [34], 2) energetic electrons escaping from the metal surface due to multiphoton photoelectron and thermoionic emission [35], and 3) UV radiation from laser-induced solid-density plasma, if any. The above three processes may additionally contribute to air ionization besides the dominant multiphoton ionization [34] from the incident laser light. It should be emphasized that this air plasma is produced prior to the hydrodynamic motion of the ablated material from the sample that occurs on the nanosecond time scale $[36,37]$. We note that the generation of air plasma prior to the hydrodynamic motion of the ablated material has been observed in the past in picosecond laser ablation [38]. The difference between plasmas produced in air and in vacuum can clearly be seen from the plasma plume images in Figure 11, which are taken using an open-shutter camera [28]. We can see that the size of hot plasma generated following ablation in air is larger than that in vacuum. Since material ejection in vacuum is not 
constrained by ambient gas, plume formed in vacuum should have a larger size than that in air if they are formed only by the ejected metal particles. The fact that we see a larger plume size in air rather than in vacuum indicates that there must be air plasma generated by a high-intensity laser pulse. The formation of air plasma during femtosecond pulse can enhance thermal energy coupling to a sample due to the following two pathways: 1) transferring the energy stored in the air plasma to the sample [34], 2) enhanced re-deposition of the ablated material back onto the sample due to the presence of a high-pressure on the sample surface from the ambient gas plasma long after the femtosecond laser pulse. The decay time of the air plasma pressure, $\tau$, is given by [39] $\tau=r / a_{p}$, where $r$ is the radius of the irradiated spot and $a_{p}$ is the sound speed in the plasma. For our ablation experiment in air, we estimate $\tau$ to be about $10 \mathrm{~ns}$ with $r=50 \mu \mathrm{m}$ and $a_{p}=5 \mathrm{~km} / \mathrm{s}$ [39]. In our experiment, we observe a greater amount of particle re-deposition within the ablation spot in air following a single pulse ablation, see e.g., SEM images in Figures 3(c) and 6(c). From the SEM images in Figure 12, we again see a greater amount of material re-deposition around the crater in air compared to that in vacuum following multi-pulse ablation. Therefore, an enhanced re-deposition of the ablated materials in air following both single-pulse and multi-pulse ablation is confirmed experimentally.

By taking into account the above-mentioned enhanced thermal coupling assisted by air plasma, the behavior of REC in Figure 2 can be explained as follows. For the low $N(N<12)$ where REC decreases from 0.76 after

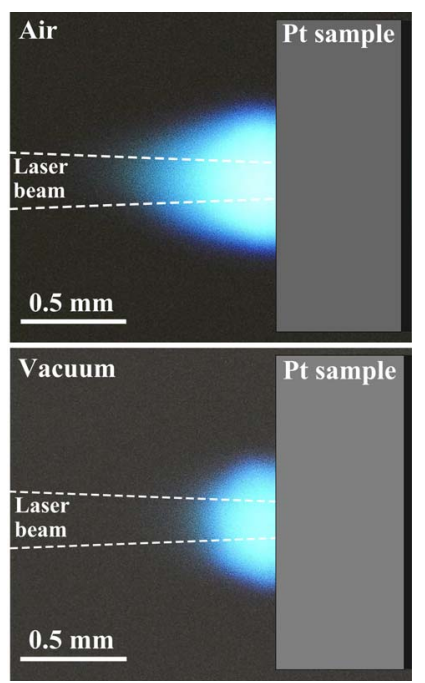

Figure 11. Open-shutter photographs of the plasma generated by single-pulse ablation at $F=3.3 \mathrm{~J} / \mathrm{cm}^{2}$ in 1 -atm air and vacuum $\left(P=7 \times 10^{-3}\right.$ Torr $)$. The laser beam is incident normally onto the sample from left.
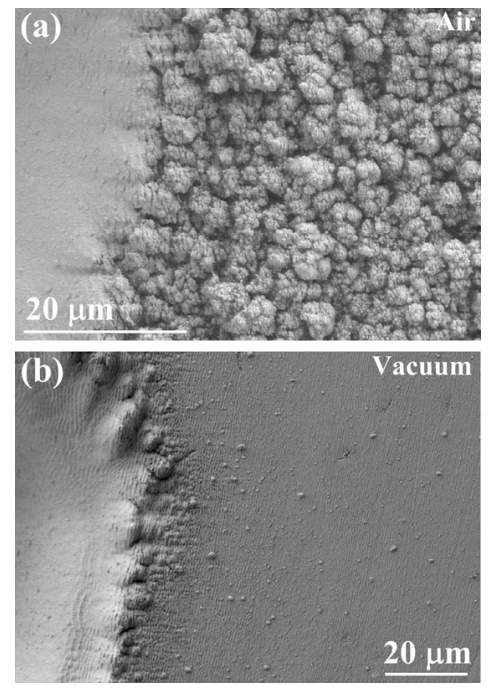

Figure 12. SEM images of the re-deposited materials on a platinum sample following 1500-pulse ablation at $F=3.3 \mathrm{~J} / \mathrm{cm}^{2}$ in (a) air and (b) vacuum. There is clearly more material re-deposition around the crater in air than in vacuum.

the first pulse to 0.65 after 12 pulses, laser ablation mainly produces surface roughness (Figure 3) and no crater is formed. This surface roughness will diffuse the surface backscattering, reduce the amount of reflected light absorbed by air plasma, and subsequently reduce the air plasma-assisted heating of the sample. For $N>12$, a cavity starts to develop (Figure 4(a)), and this causes REC to increase with $N$. The cavity effects on the enhanced REC can be more clearly seen from the REC data in vacuum (Figure 5) where the air plasma effects are excluded: we can see from the figure that, for $N<10$, REC is very low $(K \sim 0.15)$ in vacuum without the contribution of air plasma, but increases significantly with $N$ for $N>10$ when a cavity is formed (Figure 4(b)). The results obtained here show a significant effect of the reflected light on air plasma. However, a full understanding of this effect requires further experimental and theoretical studies.

\section{CONCLUSIONS}

In this paper, we perform a shot-to-shot detailed study on how residual thermal coupling depends on the absorptance change due to laser-induced surface structural modifications. Surprisingly, our study shows that, with a small number of applied pulses in air, the enhanced residual thermal coupling following the first-pulse ablation decreases with pulse number despite of an enhanced absorptance. Both residual thermal coupling and absorptance increase with pulse number following a large number of applied pulses. To explain our observation at 
a low pulse number, we propose a plasma-assisted mechanism for femtosecond laser ablation in a gas medium. Supported by our experimental results, we believe that the formation of ambient gas plasma enhances thermal energy coupling to a sample due to the following two pathways: energy transfer from the air plasma to the sample and enhanced re-deposition of hot ablated particles back onto the sample due to the presence of high pressure from the ambient gas plasma. These plasma-assisted effects can only play a role in a gas medium and are most important following a relatively low number of applied shots when only surface roughness but not a crater develops. At a large number of applied pulses, the residual heating of the sample is mainly governed by the formation of craters that enhance thermal energy coupling to the sample both in air and in vacuum. Although further studies are needed to better understand the physical mechanisms of thermal energy coupling to solids, our study provides new guidelines to determine the optimal thermal-loading conditions for achieving high-quality material processing using femtosecond laser pulses.

\section{ACKNOWLEDGMENTS}

The authors acknowledge R. Grzegorzak for assistance with vacuum equipment. The research was supported by the National Science Foundation and US Air Force Office of Scientific Research.

\section{REFERENCES}

[1] Pronko, P.P., Dutta, S.K., Squier, J., Rudd, J.V., Du, D. and Mourou G. (1995) Machining of sub-micron holes using a femtosecond laser at $800 \mathrm{~nm}$. Optics Communications, 114, 106-110.

doi:10.1016/0030-4018(94)00585-I

[2] Chichkov, B. N., Momma, C., Nolte, S., von Alvensleben, F. and Tunnermann, A. (1996) Femtosecond, picosecond and nanosecond laser ablation of solids. Applied Physics $A$, 63, 109-115. doi:10.1007/BF01567637

[3] Pereira, A., Cros, A., Delaporte, P., Georgiou, S., Manousaki, A., Marine, W. and Sentis, M. (2004) Surface nanostructuring of metals by laser irradiation: Effects of pulse duration, wavelength and gas atmosphere. Applied Physics A, 79, 1433-1437. doi:10.1007/s00339-004-2804-x

[4] Nolte, S., Chichkov, B.N., Welling, H., Shani, Y., Liebermann, K. and Terkel, H. (1999) Nanostructuring with spatially localized femtosecond laser pulses. Optics Letters, 24, 914-916. doi:10.1364/OL.24.000914

[5] Amoruso, S., Ausanio, G., Bruzzese, R., Vitello, M. and Wang, X. (2005) Femtosecond laser pulse irradiation of solid targets as a general route to nanoparticle formation in a vacuum. Physical Review B, 71, 033406. doi:10.1103/PhysRevB.71.033406

[6] Koch, J., Korte, F., Bauer, T., Fallnich, C., Ostendorf, A. and Chichkov, B.N. (2005) Nanotexturing of gold films by femtosecond laser-induced melt dynamics. Applied
Physics A, 81, 325-328.

doi:10.1007/s00339-005-3212-6

[7] Vorobyev, A.Y. and Guo, C. (2006) Femtosecond laser nanostructuring of metals. Optics Express, 14, 2164-2169. doi:10.1364/OE.14.002164

[8] Wu, C., Crouch, C.H., Zhao, L., Carey, J.E., Younkin, R., Levinson, J.A., Mazur, E., Farrell, R.M., Gothoskar, P. and Karger, A. (2001) Near-unity below-band-gap absorption by microstructured silicon. Applied Physics Letters, 78, 1850-1852. doi:10.1063/1.1358846

[9] Vorobyev, A.Y. and Guo, C. (2005) Enhanced absorptance of gold following multi-pulse femtosecond laser ablation. Physical Review B, 72, 195422. doi:10.1103/PhysRevB.72.195422

[10] Vorobyev, A.Y. and Guo, C. (2008) Colorizing metals with femtosecond laser pulses. Applied Physics Letters, 92, 041914. doi:10.1063/1.2834902

[11] Kaakkunen, J.J.J., Paivasaari, K., Kuittinen, M. and Jaaskelainen, T. (2009) Morphology studies of the metal surfaces with enhanced absorption fabricated using interferometric femtosecond ablation. Applied Physics A, 94, 215-220. doi:10.1007/s00339-008-4895-2

[12] Paivasaari, K., Kaakkunen, J.J.J., Kuittinen, M. and Jaaskelainen, T. (2007) Enhanced optical absorptance of metals using interferometric femtosecond ablation. $O p$ tics Express, 15, 13838-13843. doi:10.1364/OE.15.013838

[13] Ausano, G., Barone, A.C., Iannotti, V., Lanotte, L., Amoruso, S., Bruzzese, R. and Vitiello M. (2004) Magnetic and morphological characteristics of nickel nanoparticles films produced by femtosecond laser ablation. Applied Physics Letters, 85, 4103-4105. doi:10.1063/1.1815065

[14] Baldacchini, T., Carey, J.E., Zhou, M. and Mazur, E. (2006) Superhydrophobic Surfaces Prepared by Microstructuring of Silicon Using a Femtosecond Laser. Langmuir, 22, 4917-4919. doi:10.1021/la053374k

[15] Zorba, V., Persano, L., Pisignano, D., Athanassiou, A., Stratakis, E., Cingolani, R., Tzanetakis, P. and Fotakis, C. (2006) Making silicon hydrophobic: Wettability control by two-lengthscale simultaneous patterning with femtosecond laser irradiation. Nanotechnology, 17, 3234-3238. doi:10.1088/0957-4484/17/13/026

[16] Vorobyev, A.Y. and Guo, C. (2009) Metal pumps liquid uphill. Applied Physics Letters, 94, 224102. doi:10.1063/1.3117237

[17] Fadeeva, E., Schlie, S., Koch, J., Chichkov, B.N., Vorobyev, A.Y. and Guo, C. (2009) Femtosecond laser-induced surface structures on platinum and their effects on hydrophobicity and fibroblast cell proliferation. Contact Angle, Wettability and Adhesion, 6, 163-171.

[18] Qian, F., Cracuin, V., Singh, R.K., Dutta, S.D. and Pronko, P.P. (1999) High intensity femtosecond laser deposition of diamond-like carbon films. Journal of Applied Physics, 86, 2281-2290. doi:10.1063/1.371043

[19] Luke, J.R., Phipps, C.R. and McDuff, G.G. (2003) Laser plasma thruster. Applied Physics A, 77, 343-348.

[20] Polte, T.R., Shen, M., Karavitis, J., Montoya, M., Pendse, J., Xia, S., Mazur, E. and Ingber, D.E. (2007) Nanostructured magnetizeable materials that switch cells between life and death. Biomaterials, 28, 2783-2790. doi:10.1016/j.biomaterials.2007.01.045

[21] Anisimov, S.I. and Luk'yanchuk, B.S. (2002) Selected 
problems of laser ablation theory. Physics-Uspekhi, 45, 293-324. doi:10.1070/PU2002v045n03ABEH000966

[22] Stoian, R., Rosenfeld, A., Ashkenasi, D., Hertel, I.V., Bulgakova, N.M. and Campbell, E.E.B. (2002) Surface charging and impulsive ion ejection during ultrashort pulsed laser ablation. Physical Review Letters, 88, 097603. doi:10.1103/PhysRevLett.88.097603

[23] Bulgakova, N.M., Stoian, R., Rosenfeld, A., Hertel, I.V. and Campbell, E.E.B. (2004) Electronic transport and consequences for material removal in ultrafast pulsed laser ablation of materials. Physical Review B, 69, 054102. doi:10.1103/PhysRevB.69.054102

[24] Ivanov, D.S. and Zhigilei, L.V. (2003) Combined atomistic-continuum modeling of short-pulse laser melting and disintegration of metal films. Physical Review B, $6 \mathbf{8}$, 064114. doi:10.1103/PhysRevB.68.064114

[25] Zhigilei, L.V., Lin, Z. and Ivanov, D.S. (2009) Atomistic Modeling of Short Pulse Laser Ablation of Metals: Connections between Melting, Spallation, and Phase Explosion. The Journal of Physical Chemistry C, 113, 1189211906. doi:10.1021/jp902294m

[26] Povarnitsyn, M.E., Itina, T.E., Sentis, M., Khishchenko, K.V. and Levashov, P.R. (2007) Material decomposition mechanisms in femtosecond laser interactions with metals. Physical Review B, 75, 235414. doi:10.1103/PhysRevB.75.235414

[27] Gamaly, E.G., Rode, A.V., Luther-Davies, B. and Tichonchuk, V.T. (2002) Ablation of solids by femtosecond lasers: Ablation mechanism and ablation thresholds for metals and dielectrics. Physics of Plasmas, 9, 949-957. doi:10.1063/1.1447555

[28] Vorobyev, A.Y., Kuzmichev, V.M., Kokody, N.G., Kohns, P., Dai, J. and Guo, C. (2006) Residual thermal effects in Al following single ns- and fs-laser pulse ablation. $A p$ plied Physics A, 82, 357-362. doi:10.1007/s00339-005-3412-0

[29] Vorobyev, A.Y. and Guo, C. (2005) Direct observation of enhanced residual thermal energy coupling to solids in femtosecond laser ablation. Applied Physics Letters, 86, 011916. doi:10.1063/1.1844598

[30] Kaye, G.W.C. and Laby, T.H. (1956) Tables of Physical and Chemical Constants. 11th Edition, Longmans, Lon- don.

[31] McKay, J. A., Bleach, R. D., Nagel, D. J., Schriemph, J. T., Hall, R. B., Pond, C. R. and Manlief, S. K. (1979) Pulsed- $\mathrm{CO}_{2}$-laser interaction with aluminum in air: Thermal response and plasma characteristics. Journal of Applied Physics, 50, 3231-3240. doi:10.1063/1.326361

[32] Vorobyev, A.Y. (1985) Reflection of the pulsed ruby laser radiation by a copper target in air and in vacuum. Soviet Journal of Quantum Electronics, 15, 490-493. doi:10.1070/QE1985v015n04ABEH006963

[33] Milchberg, H. M., Clark, T. R., Durfee, C. G., Antonsen, T. M. and Mora, P. (1996) Development and applications of a plasma waveguide for intense laser pulses. Physics Plasmas, 3, 2149-2155. doi:10.1063/1.871668

[34] Bulgakova, N.M., Zhukov, V.P., Vorobyev, A.Y. and Guo, C. (2008) Modeling of residual thermal effect in femtosecond laser ablation of metals. role of gas environment. Applied Physics A, 92, 883-889. doi:10.1007/s00339-008-4568-1

[35] Fujimoto, J. G., Liu, J.M. and Ippen, E.P. (1984) Femtosecond Laser interaction with Metallic Tungsten and Nonequilibrium Electron and Lattice Temperatures. $\mathrm{Ap}$ plied Physics Letters, 53, 1837-1840. doi:10.1103/PhysRevLett.53.1837

[36] König, J., Nolte, S. and Tünnermann, A. (2005) Plasma evolution during metal ablation with ultrashort laser pulses. Optics Express, 13, 10597-10607. doi:10.1364/OPEX.13.010597

[37] Breitling, D., Ruf, A., Berger, P.W., Dausinger, F.H., Klimentov, S.M., Pivovarov, P.A., Kononenko, T.V. and Konov, V.I. (2003) Plasma effects during ablation and drilling using pulsed solid-state lasers. Proceedings of SPIE, 5121, 24-33. doi: $10.1117 / 12.513766$

[38] Mao, S.S., Mao, X., Greif, R. and Russo, R.E. (2000) Dynamics of an air breakdown plasma on a solid surface during picosecond laser ablation. Applied Physics Letters, 76, 31-33. doi:10.1063/1.125646

[39] McKay, J.A., Schriemph, J.T., Cronburg, T.L., Eninger, J.E. and Woodroffe, J.A. (1980) Pulsed $\mathrm{CO}_{2}$ laser interaction with a metal surface at oblique incidence. Applied Physics Letters, 36, 125-127. doi:10.1063/1.91403 